Schweizerisches Jahrbuch für Entwicklungspolitik

$19 \mid 2000$

Für eine bessere Kohärenz der schweizerischen

Beziehungen mit den Entwicklungsländern

\title{
Leitbild Nord-Süd : ein neuer Anlauf
}

Thierry Pellet

\section{(2) OpenEdition \\ Journals}

Electronic version

URL: http://journals.openedition.org/sjep/777

DOI: 10.4000/sjep.777

ISSN: 1663-9677

Publisher

Institut de hautes études internationales et du développement

\section{Printed version}

Date of publication: 1 avril 2000

Number of pages: 115-120

ISSN: 1660-5926

\section{Electronic reference}

Thierry Pellet, "Leitbild Nord-Süd : ein neuer Anlauf", Schweizerisches Jahrbuch für Entwicklungspolitik [Online], 19 | 2000, Online erschienen am: 23 August 2012, abgerufen am 11 März 2021. URL: http:// journals.openedition.org/sjep/777 ; DOI: https://doi.org/10.4000/sjep.777 


\section{LEITBILD NORD-SÜD: EIN NEUER ANLAUF}

THIERRY PELLeT*

D

AS 1994 VERÖFFENTLICHTE LEITBILD NORD-SüD löste in den Kreisen der Entwicklungspolitik ein nachhaltiges Echo aus. Von den einen wegen seines innovativen Ansatzes begrüsst, von anderen wiederum aufgrund seiner Schwachstellen kritisiert, sandte dieses Dokument dennoch ein deutliches Signal dafür aus, dass künftig den Worten auch Taten folgen sollten. Sechs Jahre nach seiner Veröffentlichung wird an dieser Stelle eine Standortbestimmung vorgenommen, und zwar aus der Sicht einer in der Entwicklungspolitik tätigen Organisation wie der Erklärung von Bern, die sich in ihrer täglichen Arbeit immer wieder mit der Frage der Kohärenz in den aussenpolitischen Beziehungen der Schweiz auseinandersetzt.

\section{ENTSTEHUNSGESCHICHTE}

Als Einstieg sollen einige Fakten in Erinnerung gerufen werden, die den Zeitraum zwischen der Erarbeitung des Leitbildes 1990 bis zu seiner Veröffentlichung 1994 massgeblich prägten.

Erstmals wurde in der Schweiz ein ehrgeiziger Schritt unternommen, um die verschiedenen Aspekte der helvetischen Aussenpolitik, die bis anhin teilweise miteinander im Widerspruch standen, zu harmonisieren. Zentrales Element war dabei das Streben nach Kohärenz, die «nicht mehr länger nur die Entwicklungszusammenarbeit betrifft, sondern die Gesamtheit der Beziehungen der Schweiz mit den Entwicklungsländern »1 .

In Bern schien ein neuer Wind zu wehen, der nicht zuletzt von der allgemeinen Aufbruchstimmung herrührte, die seit dem Erdgipfel von Rio über die nachhaltige Entwicklung (Konferenz der Vereinten Nationen für Umwelt und Entwicklung, UNCED) herrschte. Die visionäre Botschaft, wonach «unser langfristiges Wohlergehen auch vom Schicksal des Südens abhängt», fiel auf fruchtbaren Boden, zumal die Stimmung in der Schweiz noch immer vom Optimismus, der auf dem Wirtschaftswachstum der 80er Jahre gründete, geprägt war². Alle Anzeichen wiesen darauf hin, dass der Zeitpunkt für eine Öffnung des Landes und für eine Verstärkung der Kohärenz günstig war.

Der Bundesrat nutzte die vorteilhaften Umstände und legte einen starken politischen Willen an den Tag, unter der Beteiligung mehrerer Departemente einen ambitiösen Text auszuarbeiten. Es folgten zähe Verhandlungen und zahllose Rücküberweisungen des Entwurfs von einem Bundesamt ins andere, die dazu

* Ökonom, ständiger Sekretär der Erklärung von Bern.

1. Bericht des Bundesrates über die Nord-Süd-Beziehungen der Schweiz in den 90er Jahren (Leitbild Nord-Süd) vom 7. März 1994, DEZA, Bern, 1994. Das Leitbild kann auf der Website der DEZA konsultiert werden: www.deza.admin.ch (Link: Entwicklungspolitik, Grundlagen).

2. Zwar wurde in der Schweiz bereits zu Beginn der 90er-Jahre ein Ansteigen der Inflation und der Arbeitslosigkeit verzeichnet, die Tragweite und die Dauer der Krise wurden jedoch erst später bewusst wahrgenommen. 
führten, dass Fristen nicht eingehalten und die Veröffentlichung des Leitbildes verzögert wurden. An der Ausarbeitung des Leitbildes waren zwei Departemente massgeblich beteiligt: Das Eidgenössische Departement für auswärtige Angelegenheiten (EDA) mit seiner Direktion für Entwicklungszusammenarbeit und humanitäre Hilfe (DEH, heute DEZA) unter der Leitung von Flavio Cotti, und das Eidgenössische Volkswirtschaftsdepartement (EVD) mit seinem Bundesamt für Aussenwirtschaft (BAWI, heute seco), welches von Jean-Pascal Delamuraz geführt wurde. Diese beiden Bundesämter, die sich sonst eher argwöhnisch gegenüber stehen, waren aufgerufen, das Leitbild gemeinsam zu erarbeiten.

\section{DER START}

Nicht zuletzt dank der politischen Legitimität, die aufgrund der langwierigen Konsenssuche ausser Frage steht, besitzt das Leitbild Nord-Süd Referenzcharakter. Nachdem die Erarbeitung des Dokumentes erhebliche Zeit beansprucht hatte, stiess es bei den Nichtregierungsorganisationen (NRO) und den Medien auf lebhaftes Interesse, wodurch sich der Druck, den Text auch zu respektieren, noch erhöhte.

Für die Erklärung von Bern stellte das Leitbild eine willkommene Ergänzung zu den beiden Gesetzestexten dar, die bislang als Grundlage für ihre Bemühungen zur Förderung der Kohärenz gedient hatten: dem Bundesgesetz über die internationale Entwicklungszusammenarbeit und die humanitäre Hilfe (1976) und dem Bundesgesetz über die Exportrisikogarantie (1958, revidiert 1981 und 1996). Im ersteren ist insbesondere festgehalten, die Entwicklungszusammenarbeit sei langfristig auf «besser ausgewogene Verhältnisse in der Völkergemeinschaft» (1976, Art. 5) ausgerichtet, wohingegen im zweiteren präzisiert wird, dass «bei Exporten nach ärmeren Entwicklungsländern [der Bund] die Grundsätze der schweizerischen Entwicklungspolitik mitzuberücksichtigen» habe (1981, Art. 1, Abs. 2). Gegenüber diesen beiden Gesetzestexten besass das Leitbild Nord-Süd aus formaler Sicht zweifellos keinen besonders verbindlichen Charakter ${ }^{3}$. Dieses Defizit wurde jedoch durch das politische Gewicht der Botschaft kompensiert. Für eine Beurteilung des Gehalts und der Praxistauglichkeit des Leitbildes war jedoch gemäss der damals vorherrschenden Meinung ein gewisser zeitlicher Abstand notwendig.

Seither hat sich die Erklärung von Bern mit mehreren Dossiers befasst, die mit der Frage nach der Kohärenz in der schweizerischen Aussenpolitik im Zusammenhang standen. Typische Fälle betrafen die Gewährung von Exportrisikogarantien (ERG) an Schweizer Unternehmen durch den Bund, zumal diese Entscheidungen an der Schnittstelle zwischen dem privaten und dem öffentlichen Sektor ansetzen. Beispiele dafür wären etwa das Drei-Schluchten-Projekt in China $^{4}$ oder der Staudamm von Ilisu in der Türkei ${ }^{5}$.

3. Zur Erinnerung: Ursprünglich sollte das Leitbild als Anhang zum Bericht des Bundesrates über die Aussenpolitik der Schweiz in den 90er Jahren (1993) veröffentlicht werden; dieses Vorhaben wurde schliesslich jedoch aufgegeben.

4. ABB erhielt 1997 eine erste ERG in der Höhe von 240 Millionen Franken, die 1999 durch eine zweite über 160 Millionen Franken ergänzt wurde.

5. 1998 erhielt Sulzer eine ERG über 130 Millionen Franken. 
Das wohl schlagendste Beispiel für diesen Sachverhalt ist zweifellos das DreiSchluchten-Projekt. In kaum einem anderen Fall sind die Divergenzen zwischen kurzfristigen privaten Interessen und langfristigen sozialen und ökologischen Anliegen der nachhaltigen Entwicklung derart krass zu Tage getreten. Im Vorfeld der symbolträchtigen Entscheidung des Bundesrates, ABB in den Genuss einer ERG kommen zu lassen, bildeten sich zwei Lager, deren Argumente einen Konsens nahezu unmöglich machten : Auf der einen Seite wiesen Entwicklungs, Umwelt- und Menschenrechtsorganisationen - deren Anliegen ebenfalls in das Leitbild Eingang gefunden hatten - auf die schwerwiegenden Probleme hin, die durch das Megaprojekt enstehen würden, und vertraten die Ansicht, der Bund könne unmöglich ein derart zerstörerisches Bauwerk mit Steuergeldern finanzieren. Ihnen gegenüber standen die Verfechter des Wirtschaftsstandortes Schweiz, die den Standpunkt vertraten, China habe ausreichende Garantien für die Lösung dieser Probleme geleistet und alle anderen Bedenken seien eine Frage der nationalen Souveränität. Im Anschluss an einen Grundsatzentscheid des Bundesrates vom Dezember 1996 entschied sich die Kommission für die Exportrisikogarantie schliesslich nach langem Zögern im August 1997 für die Gewährung einer ERG zugunsten von ABB und setzte sich damit über gewichtige Gegenargumente aus den eigenen Reihen hinweg, die insbesondere von der DEZA oder den Gewerkschaften vorgebracht worden waren. Die Arbeitsplätze und der internationale Wettbewerb (in der Zwischenzeit hatten Unternehmen in anderen Ländern bereits Exportrisikogarantien zugesichert bekommen) wogen offensichtlich schwerer als soziale und ökologische Bedenken (knapp zwei Millionen Menschen mussten zwangsweise umgesiedelt werden). Die überaus deutliche Vorgabe des Leitbildes, wonach «bei der Risikoabwägung der ERG für Lieferungen in ärmere Entwicklungsländer [...] Aspekte der [...] Achtung von Menschenrechten im Empfängerland ein zusätzliches Gewicht [erhalten] ${ }^{6}$, wurde damit klar vernachlässigt, und die relative Verbindlichkeit des Leitbildes, welches keinerlei formelle Rekursmechanismen für frappante Fälle wie diesen vorsieht, mit dieser Entscheidung offensichtlich gemacht.

Aber auch im Zusammenhang mit anderen Dossiers konnte sich die Erklärung von Bern auf das Leitbild berufen, etwa bei ihren Bemühungen für eine präventive Sperrung der Schweizer Guthaben Mobutus im Frühjahr 1997 oder der Gelder Suhartos im Juli 1998 und im Mai 1999. Damit rief sie in Erinnerung, dass der Bundesrat die Notwendigkeit des Kampfes gegen die Korruption und die Kapitalflucht sowohl auf nationaler als auch auf internationaler Ebene ausdrücklich anerkannt hatte. Eine weitere Gelegenheit, auf das Leitbild hinzuweisen, bot sich Ende 1995, als das BAWI die neue Ausrichtung der Wirtschaftsund Handelspolitik präsentierte. Mit dem Vorschlag, eine Schweizer Finanzierungsgesellschaft im Bereich der Entwicklungspolitik zu gründen, wurde eine Reihe von Fragen bezüglich der Gewichtung der öffentlichen Entwicklungshilfe gegenüber dem Privatsektor aufgeworfen. Die Erklärung von Bern wies in diesem Zusammenhang einmal mehr darauf hin, dass Erwägungen bezüglich der sozialen Gerechtigkeit bei der Planung der Wirtschafts- und Handelspolitik für die Wahrung der Kohärenz unabdingbar sind. 


\section{NACHLASSENDER ENTHUSIASMUS}

Sechs Jahre danach scheint sich der Elan verflüchtigt zu haben. Das Leitbild Nord-Süd steht heute etwas im Hintergrund, und auch der politische Wille, der schweizerischen Aussenpolitik zu verstärkter Kohärenz zu verhelfen, hat sich mittlerweile abgeschwächt. Obgleich das Dokument nichts von seiner Aktualität eingebüsst hat, wurden in den wichtigsten Bereichen wie etwa der Wirtschaftsförderung kaum Fortschritte erzielt. Mittlerweile steht gar zu befürchten, dass die wenigen Erfolge im Zuge der jüngsten Reform der Bundesverwaltung zunichte gemacht werden.

Untersucht man die Vergabe von Exportrisikogarantien für grosse Staudämme, so scheint sich diese Befürchtung zu bestätigen. Zwar fand in den vergangenen Jahren eine durchaus positive Entwicklung statt: Nach einer lebhaften öffentlichen Debatte (Drei-Schluchten-Staudamm und Gewährung einer ersten ERG zugunsten von ABB im August 1997) wurden erstmals verschiedene NRO von der ERG-Kommission angehört (Staudamm von Ilisu, ERG für Sulzer im November 1998). Die Gewährung einer zweiten ERG an ABB für das DreiSchluchten-Projekt im Juni 1999 hingegen bedeutete eine klare Verschlechterung: Das Ganze spielte sich ohne Anhörung und unter absoluter Geheimhaltung $\mathrm{ab}$. Ob es sich dabei lediglich um ein ungeschicktes Vorgehen oder um einen regelrechten Rückschritt handelt, wird die Zukunft erweisen.

Der nachlassende Enthusiasmus ist auf mehrere Ursachen zurückzuführen:

- Angesichts des nicht verbindlichen Charakters des Leitbildes Nord-Süd wird der politische Wille der Exekutive zum entscheidenden Faktor. Vier der 1994 amtierenden Bundesräte und insbesondere die Vorsteher der beiden am stärksten implizierten Departemente, Jean-Pascal Delamuraz und Flavio Cotti, sind nicht mehr im Amt. Zwar verweist der derzeitige Vorsteher des EDA, Joseph Deiss, immer wieder auf die Notwendigkeit einer verstärkten Kohärenz, so auch anlässlich seiner Afrika-Reise im Oktober 1999. Nach wie vor haben jedoch die beiden Bundesräte Couchepin und Deiss ihren Willen, die Kohärenz in der Praxis umzusetzen, noch nicht unter Beweis gestellt.

๖ Die jüngsten Ereignisse bestätigen diese Einschätzung: Mit der Errichtung des Staatssekretariats für Wirtschaft (seco) innerhalb des Eidgenössischen Volkswirtschaftsdepartementes (EVD) wurden das BAWI und das Bundesamt für Wirtschaft und Arbeit (BWA) in einer organisatorischen Einheit zusammengefasst. Das BAWI, welches rund ein Drittel der öffentlichen Entwicklungshilfe der Schweiz abwickelt, befindet sich nunmehr innerhalb einer weitverzweigten organisatorischen Struktur, in der kurzfristige wirtschaftliche Interessen in den Entscheidungen überwiegen dürften. Mit der Schaffung des seco wurde ein weiteres Projekt, welches in der Vergangenheit ebenfalls zur Debatte gestanden hatte, endgültig ad acta gelegt, nämlich die Zusammenlegung der Direktion für Entwicklung und Zusammenarbeit (DEZA) und des BAWI.

- Ein weiterer Grund dafür, dass das Konzept der Kohärenz in den 90er Jahren nicht weiter entwickelt wurde, ist zweifellos in der Verschlechterung der schweizerischen Wirtschaftslage zu suchen. Angesichts der allgemeinen 
Krise und der anhaltenden Arbeitslosigkeit ${ }^{7}$ erhielten kurzfristige nationale Interessen in den Debatten und bei der Abwägung der verschiedenen entwicklungspolitischen Ziele ein grösseres Gewicht. Im Laufe des intensiven Lobbyings, das ABB 1997 für die Gewährung einer ERG für den DreiSchluchten-Staudamm durchführte, verkam der wirtschaftliche Druck endgültig zur Erpressung, bei der mit dem Verlust von Arbeitsplätzen gedroht wurde. Gegenüber der von ABB in Aussicht gestellten Auslagerung von 170 Arbeitsplätzen in andere Länder wie etwa Deutschland über einen Zeitraum von sechs Jahren hatten soziale und Umweltanliegen wenig Gewicht.

- Auch der zeitliche Abstand forderte seinen Tribut. Während seit 1994 die Mehrheit der Bundesräte ausgewechselt wurde, fanden im gleichen Zeitraum gleich zweimal National- und Ständeratswahlen statt (1995 und 1999). Bereits 1996 wies Thomas Greminger (DEZA) darauf hin, das Parlament habe das Leitbild zwar positiv aufgenommen, die Debatte aber bislang nicht weiter geführt, und warf die Frage auf, ob die Bedeutung des Konzeptes der Kohärenz auch wirklich auf allen Seiten erkannt worden sei ${ }^{8}$. Die zweifache Erneuerung des Parlaments dürfte dem Verständnis nicht unbedingt zuträglich gewesen sein. Damit obliegt es allein der Exekutive, den Grundsätzen des Leitbildes auch in Zukunft Geltung zu verschaffen.

Wie auch Jacques Forster in seinem Beitrag zum Dossier dieser Ausgabe darlegt ${ }^{9}$, werden die bei der Anwendung des Kohärenzprinzips entstehenden Interessenskonflikte aufgrund des Kräfteverhältnisses unter den Beteiligten gelöst. Angesichts der verschlechterten wirtschaftlichen Lage muss leider festgestellt werden, dass die derzeitigen Kräfteverhältnisse der Suche nach der Kohärenz weniger förderlich sind als vor sechs Jahren. In ihrer täglichen Arbeit muss die Erklärung von Bern immer wieder feststellen, dass der Schutz von Arbeitsplätzen in den heutigen Entscheidungen eine grössere Rolle spielt als in der Vergangenheit.

\section{EIN NEUER ANLAUF}

Um die 1994 mit viel Enthusiasmus begonnene Suche nach der Kohärenz steht es heute eher schlecht. Einzig ein energischer politischer Schritt wie jener, der die Ausarbeitung des Leitbildes überhaupt erst möglich gemacht hat, kann dem ambitiösen Konzept der Kohärenz zu neuer Legitimität verhelfen. Dabei ist in erster Linie der Bundesrat angesprochen, der, ähnlich wie 1994, ein unmissverständliches Zeichen setzen muss. Damit könnte eine neue Debatte lanciert werden, bei der die Anwendbarkeit der im Leitbild festgehaltenen Grundsätze vermehrt ins Zentrum gerückt werden muss. Dies wäre eine weitere Gelegenheit, sämtliche Bereiche der Bundesverwaltung einzubeziehen und auch die Wissenschaft, die NRO und nicht zuletzt die breite Öffentlichkeit in die Diskussion einzubinden.

7. Der 1999 beobachtete wirtschaftliche Aufwärtstrend, sei er nun konjunkturell oder strukturell bedingt, hat sich noch nicht überall bemerkbar gemacht. Gerade in diesem Dossier von globaler Bedeutung herrscht eine besonders ausgeprägte Schwerfälligkeit.

8. T. Greminger, «La cohérence des politiques - un premier bilan de la mise en œuvre des Lignes directrices NordSud de la Suisse», in D. Fino, Impasses et promesses. L'ambiguité de la coopération au développement, Nouveaux Cahiers de l'IUED, Nr. 4, Paris/Genève, PUF/IUED, 1996, S. 146.

9. J. Forster, «Politikkohärenz: Ein neuer Ansatz der Nord-Süd-Beziehungen », im Dossier dieser Ausgabe des Jahrbuchs. 
In diesem Prozess könnte unter Umständen ein neuer, das Leitbild ergänzender Text erarbeitet werden. Allerdings müsste dem Dokument ein anderer Stellenwert unter den Texten des Bundes eingeräumt und seine formelle Legitimität geklärt werden, um zu verhindern, dass ihm das gleiche Schicksal wie dem Leitbild widerfährt. Vor allem jedoch böte sich damit die Gelegenheit, umweltpolitische und soziale Prinzipien - etwa im Hinblick auf die Zwangsumsiedlung von Bevölkerungsgruppen - in den Text einfliessen zu lassen, welche von der Exportindustrie beachtet werden müssen. Wie dies zu bewerkstelligen ist, hat die Weltbank bereits vorgemacht. Ebenfalls nötig sind formelle Mechanismen mit bindendem Charakter, Konsultationsverfahren, Prinzipien bezüglich der Transparenz sowie Rekursmechanismen. Schliesslich müsste dem Dokument auch auf internationaler Ebene Geltung verschafft werden. Ein Weg dazu wäre die aktive Teilnahme an der Ausarbeitung und Harmonisierung von Standards im sozialen und Umweltbereich, welche von den Exportkredite gewährenden Stellen der OECD-Länder umgesetzt werden müssen.

Zwar muss der erste Schritt vor allem von den beiden Bundesräten Couchepin und Deiss ausgehen. Aber auch das Parlament und die Zivilgesellschaft sind aufgerufen, aktiv zu werden, um den Motor wieder in Gang zu setzen. Wie dringend der Handlungsbedarf ist, wird angesichts des sich verschärfenden Paradoxes unmissverständlich klar: Die Notwendigkeit der Kohärenz, die 1994 in der Schweiz unbestritten war, scheint zunehmend an Bedeutung zu verlieren, gleichzeitig aber nimmt die gegenseitige Abhängigkeit der Länder im Zuge der fortschreitenden Globalisierung zu. Wichtige Entscheidungen wie die Gründung der Welthandelsorganisation im Jahre 1995 oder die rasante Entwicklung der Telekommunikation haben aber dazu geführt, dass das Bedürfnis nach Kohärenz mittlerweile akut geworden ist. In verschiedenen Gremien wie etwa in der OECD, der Weltbank oder der Internationalen Finanzkorporation ist die Debatte über eine verstärkte Kohärenz mittlerweile so weit gediehen, dass erste Massnahmen im sozialen Bereich und auf den Gebieten der Menschenrechte und des Umweltschutzes ergriffen werden. Die OECD beispielsweise vertritt folgenden Standpunkt: «Unser oberstes Ziel muss es sein, sicherzustellen, dass die Politiken der Industrieländer auf diesem Gebiet miteinander kompatibel sind und die Verwirklichung der Zielsetzungen im Bereich der Entwicklung nicht beeinträchtigen. $\gg^{10}$ Die Schweiz läuft Gefahr, eines Tages den Anschluss zu verpassen.

Damit die linke Hand in Zukunft weiss, was die rechte tut und das Streben nach Kohärenz mehr als ein vorübergehendes Phänomen ${ }^{11}$ ist, muss ein neuer Anlauf genommen werden.

Das Leitbild für das erste Jahrzehnt des dritten Jahrtausends darf mit Spannung erwartet werden. 